\title{
Optical propagation analysis in photobioreactor measurements on cyanobacteria
}

\author{
F. Fanjul-Vélez*, J. L. Arce-Diego* \\ Applied Optical Techniques Group, TEISA Department, University of Cantabria \\ Av de los Castros s/n, 39005 Santander (Spain)
}

\begin{abstract}
Biotechnology applications are nowadays increasing in many areas, from agriculture to biochemistry, or even biomedicine. Knowledge on biological processes is becoming essential in order to be able to adequately estimate and control the production of these elements.

Cyanobacteria present the capability of producing oxygen and biomass, from $\mathrm{CO}_{2}$ and light irradiation. Therefore, they could be fundamental for human subsistence in adverse environments, as basic needs of breathing and food would be guaranteed. Cyanobacteria cultivation, as other microorganisms, is carried out in photo-bioreactors. The adequate design of photobioreactors greatly influences elements production throughput. This design includes optical illumination and optical measurement of cyanobacteria growth. In this work an analysis of optical measurement of cyanobacteria growth in a photobioreactor is made. As cyanobacteria are inhomogeneous elements, the influence of light scattering is significant. Several types of cyanobacteria are considered, as long as several spatial profiles and irradiances of the incident light. Depending on cyanobacteria optical properties, optical distribution of transmitted light can be estimated. These results allow an appropriate consideration, in the optical design, of the relationship between detected light and cyanobacteria growth. As a consequence, the most adequate conditions of elements production from cyanobacteria could be estimated.
\end{abstract}

Keywords: photo-bioreactor, optical propagation in tissues, cyanobacteria, light scattering, biotechnology

\section{INTRODUCTION}

Biotechnology applications are nowadays increasing in many areas, from agriculture to biochemistry, or even biomedicine. Microorganisms could be beneficial as they can supply fuel, drugs, food or even oxygen, among other products. Knowledge on biological processes is becoming essential in order to be able to adequately estimate and control the production of these elements.

Cyanobacteria are a particular type of microorganisms that present the capability of producing oxygen and biomass, from $\mathrm{CO}_{2}$ and light irradiation. Therefore, they could be fundamental for human subsistence in adverse environments, as basic needs of breathing and food would be guaranteed. Their behavior is of utmost relevance for maximizing relevant elements production [1].

Cyanobacteria cultivation, as other microorganisms, is carried out in bioreactors [2]. These devices are specifically designed to favor microorganisms growth, by supplying and controlling the elements they need. In the particular case of cyanobacteria, light irradiation is fundamental for their growth, and the devices are then called photobioreactors. The adequate design of photobioreactors greatly influences elements production throughput. This design includes optical illumination and optical measurement of cyanobacteria growth. In this work an analysis of optical measurement of cyanobacteria growth in a photobioreactor is made. As cyanobacteria are inhomogeneous elements, the influence of light scattering is significant. Several types of cyanobacteria are considered, as long as several spatial profiles and irradiances of the incident light. Depending on cyanobacteria optical properties, optical distribution of transmitted light can be estimated. These results allow an appropriate consideration, in the optical design, of the relationship between detected light and cyanobacteria growth. As a consequence, the most adequate conditions of elements production from cyanobacteria could be estimated.

*fanjulf@unican.es; arcedj@unican.es; phone +34 942206730; www.teisa.unican.es/toa

Photonics, Devices, and Systems VII, edited by Karel Fliegel, Petr Páta,

Proc. of SPIE Vol. 10603, 106030E · (c) 2017 SPIE · CCC code:

$0277-786 \mathrm{X} / 17 / \$ 18 \cdot$ doi: $10.1117 / 12.2292442$

Proc. of SPIE Vol. 10603 106030E-1 


\section{PHOTO-BIOREACTOR CHARACTERISTICS}

Photo-bioreactor design involves the consideration of several factors. Among the most relevant ones is optical irradiation. Some photo-bioreactors employ solar light, but it is difficult to control. Typically, solar light can suffer from unequal exposition to all the bacteria, low area to volume ratios, slow mass transference and inefficiency. Cyanobacteria are usually grown with LED optical sources or fluorescent lamps. Light irradiation depends on source to bioreactor distance, cyanobacteria concentration or the biofilm formation. Uneven illumination makes some cyanobacteria stop the photosynthesis process. Optical illumination spectrum is also quite relevant. Usually optimum wavelengths are in the range from 400 to $700 \mathrm{~nm}$. Irradiance also greatly influences the efficiency of the process. An insufficient irradiance results in growth decreasing, while an irradiation excess may provoke photo inhibition.

The shielding effect of surface cyanobacteria with respect to deep cyanobacteria has to be considered [3]. Photobioreactors usually comprise a stirring system to cope with this problem. Although a stirrer makes every cyanobacteria be exposed to light irradiation, it also imposes a non-continuous irradiation. This aspect should be also taken into account. $\mathrm{CO}_{2}$ supply is also needed for cyanobacteria growth. It is usually introduced in the photo-bioreactor by means of continuous injection at the bottom of the sample. The amount of $\mathrm{CO}_{2}$ supply is also a relevant parameter for the efficiency of the process. Even $\mathrm{pH}$ should be controlled in order to maintain the appropriate conditions for cyanobacteria growth.

As a summary, an adequate photo-bioreactor should show a short light irradiation trajectory, high cellular density, high light/darkness cycle, short sample size, continuous $\mathrm{CO}_{2}$ supply, constant temperature and optimal $\mathrm{pH}$.

The basis of the production process in cyanobacteria is oxygenic photosynthesis [1]. Water in the bioreactor is used as an electrons donor in combination with $\mathrm{CO}_{2}$, and oxygen $\mathrm{O}_{2}$ is freed. The photosynthesis process can be divided into two phases, the light-dependent and light-independent ones. The first phase converts light in chemical energy. Light is captured by photoactive pigments, mainly chlorophylls and carotenoids [2]. The energy generated is supplied to proteins by means of photosystem I (centered at $700 \mathrm{~nm}$ ) and photosystem II (centered at $680 \mathrm{~nm}$ ). This phase generates $\mathrm{O}_{2}$. In the dark phase the new produced substances contribute to the generation of organic compounds, such as glucose, in the Calvin cycle.

Photosynthetic efficiency is governed by irradiance and wavelength $[4,5]$, temperature (with an approximate double increase every $10^{\circ} \mathrm{C}$, until damage), $\mathrm{CO}_{2}$ concentration and $\mathrm{O}_{2}$ concentration. Cyanobacteria usually contain just chlorophyll a. This and other compounds give them a characteristic blue or green color. There are also red cyanobacteria. Typically light in visible wavelength is transferred to chlorophyll a reaction center.

The considerations exposed in this section give rise to the relevance of light irradiation on cyanobacteria. The irradiance, wavelength and spatial light distribution are of utmost importance for the efficiency of the process.

\section{RESULTS AND DISCUSSION}

The influence of optical radiation on cyanobacteria in photo-bioreactors is analyzed in this section. The first question to be addressed is the great variety of cyanobacteria morphologies that exist, from unicellular to filamentous species. This variety will greatly influence the scattering properties and, as a consequence, light distribution. Light distribution can be considered by means of a Radiation Transport equation:

$$
\hat{s} \cdot \bar{\nabla} I(r, \hat{s})=-\left(\mu_{a}+\mu_{s}\right) I(r, \hat{s})+\frac{\mu_{s}}{4 \pi} \int_{4 \pi} p\left(\hat{s} \cdot \hat{s}^{\prime}\right) I\left(r, \hat{s}^{\prime}\right) d \Omega^{\prime}
$$

In this equation $I(r, \hat{s})$ is the specific intensity. The radiation is expected to be at point $r$, and to follow the direction $\hat{s}$. The scattering events are considered according to the scattering phase function $p\left(\hat{s} \cdot \hat{s}^{\prime}\right)$. Optical radiation comes from direction $\hat{s}^{\prime}$ and is redirected to $\hat{s}$. The steady-state radiation transport equation without sources can be written as in (1), where $\mu_{a}$ and $\mu_{s}$ are the absorption and scattering coefficients, respectively.

This approach can be computed by a Monte Carlo method [6]. Optical properties, mainly absorption and scattering coefficients, anisotropy of scattering and refractive index, were obtained for different concentrations of A. cylindrica [7]. Several wavelengths were considered, particularly 405, 490, 530, 625 and $730 \mathrm{~nm}$. Three different concentration were 
taken into account, $0.202 \mathrm{Kg} / \mathrm{m}^{3}, 0.296 \mathrm{Kg} / \mathrm{m}^{3}$ y $0.431 \mathrm{Kg} / \mathrm{m}^{3}$. Optical spatial radiation was considered to be either Gaussian or top-hat [8], with a varying radius between 0.1 and $0.5 \mathrm{~cm}$.

The photo-bioreactor geometry was considered cylindrically symmetric, and a width of $1 \mathrm{~cm}$ was assumed. Figure 1 shows an example of optical spatial irradiation profiles that traverses the sample.

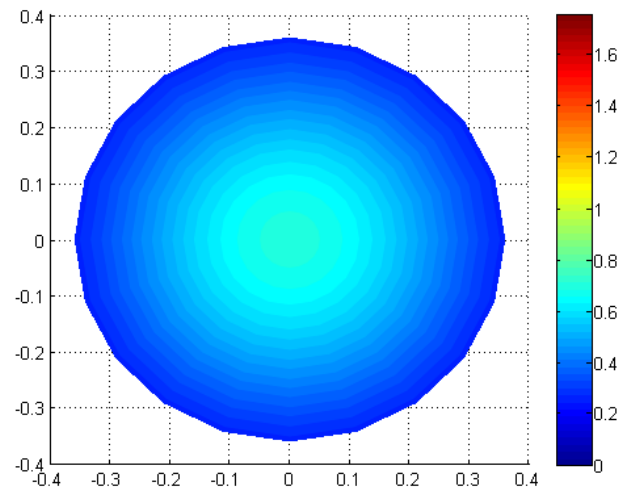

a)

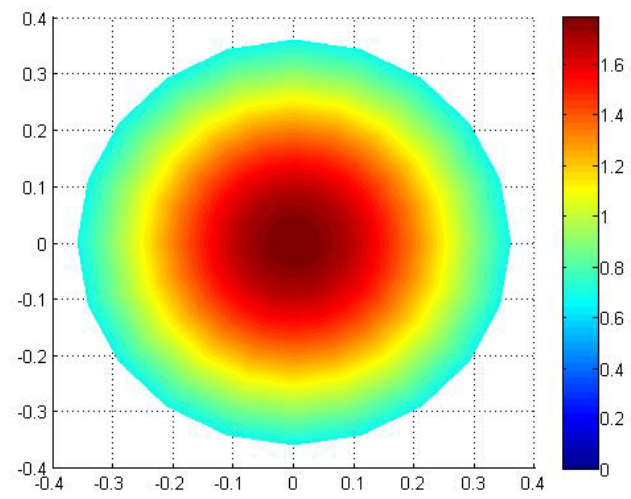

b)

Figure 1. Optical radiation distribution after traversing the photo-bioreactor filled with cyanobacteria at $0.202 \mathrm{~kg} / \mathrm{m}^{3}$ concentration, and a Gaussian $0.5 \mathrm{~cm}$ radius beam: a) $530 \mathrm{~nm}$; b) $730 \mathrm{~nm}$.

In Figure 1 the great influence of wavelength can be clearly appreciated. Optical absorption is much bigger at $530 \mathrm{~nm}$ when compared to that at $730 \mathrm{~nm}$. However, scattering coefficient at $730 \mathrm{~nm}$ makes optical radiation spread wider as in the previous case.

The evolution of the total optical fluence as a function of optical wavelength, for the case of a $0.296 \mathrm{Kg} / \mathrm{m}^{3}$ concentration can be seen in Figure 2.

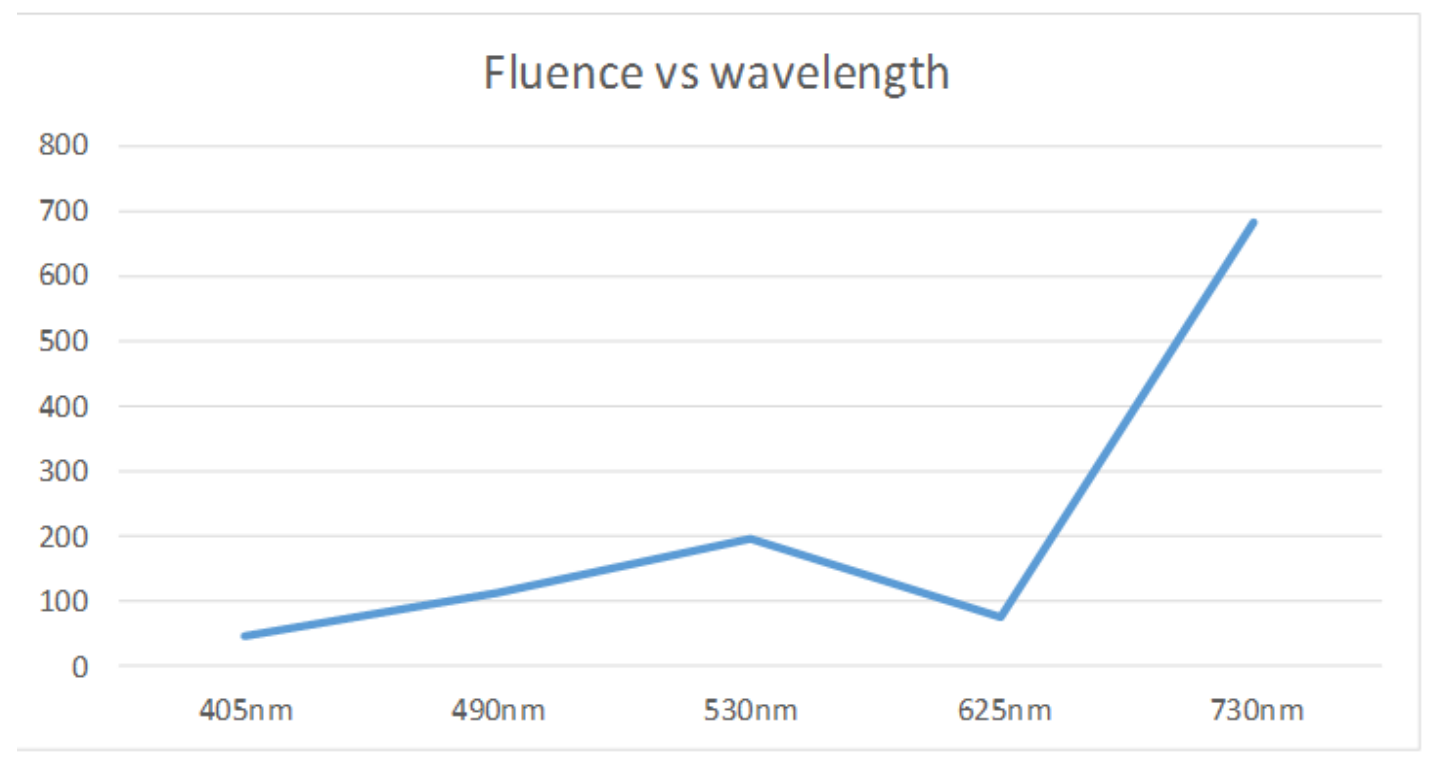

Figure 2. Optical fluence after traversing the photo-bioreactor filled with cyanobacteria at $0.296 \mathrm{~kg} / \mathrm{m}^{3}$ concentration, and a Gaussian $0.1 \mathrm{~cm}$ radius beam, as a function of wavelength. 
Figure 2 stresses the different in absorption and scattering of cyanobacteria, as a function of wavelength. In general, a decrease in wavelength is associated with an absorption increase.

\section{CONCLUSIONS}

The adequate design of photo-bioreactors is a topic of great interest in recent biotechnology. The particular case of cyanobacteria, and their production of oxygen and biomass, has been shown to be of utmost relevance to human sustainability in adverse environments. The main characteristics of cyanobacteria growth have been exposed. The work has focused on light illumination and its influence in the photosynthetic process.

Several concentrations of cyanobacteria have been considered, irradiated by optical radiation with different spatial profiles, wavelengths and radii. The results show how adequately choosing parameters such as wavelength greatly influences optical radiation distribution and, as a consequence, the photosynthetic process of each of the cyanobacteria. The results shown contribute to photo-bioreactors design and efficiency increase in cyanobacteria cultivation.

\section{ACKNOWLEDGEMENTS}

This work has been partially supported by the project "New active phases in transition metals and rare earth nano-oxides stabilized at high pressure" (MAT2015-69508-P) of the Spanish Ministry of Economy and Competitiveness, cofunded by FEDER funds, and by the San Cándido Foundation.

\section{REFERENCES}

[1] Demmig-Adams, B., "Carotenoids and photoprotection in plants. A role for the xanthophyll zeaxanthin," Biochim Biophys Acta 1020, 1-24 (1990).

[2] Wojtasiewicz, B. and Stoń-Egiert, J., "Bio-optical characterization of selected cyanobacteria strains present in marine and freshwater ecosystems," J Appl Phycol 28(4), 2299-2314 (2015).

[3] Salas-García, I., Fanjul-Vélez, F. and Arce-Diego, J. L., "Photosensitizer absorption coefficient modelling and necrosis prediction during photodynamic therapy," Journal of Photochemistry and Photobiology B: Biology 114, 79-86 (2012).

[4] Salas-García, I., Fanjul-Vélez, F. and Arce-Diego, J. L., "Superficial radially-resolved fluorescence and threedimensional photochemical time-dependent model for Photodynamic Therapy," Optics Letters 39, 1845-1848 (2014).

[5] Salas-García, I., Fanjul-Vélez, F. and Arce-Diego, J. L., "Spatial photosensitizer fluorescence emission predictive analysis for photodynamic therapy monitoring applied to a skin disease," Optics Communications 285, 1581-1588 (2012).

[6] Wang, L., Jacques, S. L. and Zheng, L., "MCML - Monte Carlo modeling of light transport in multi-layered tissues," Computer Methods and Programs in Biomedicine 47, 131-146 (1995).

[7] Heng, R. L., Lee, E. and Pilon, L., "Radiation characteristics and optical properties of filamentous cyanobacterium Anabaena cylindrica," J. Opt. Soc. Am. A31, 836-845 (2014).

[8] Fanjul-Vélez, F., Salas-García, I. and Arce-Diego, J. L., "Analysis of laser surgery in non-melanoma skin cancer for optimal tissue removal,” Laser Physics 25, 025606-1, 8 (2015). 